\title{
$\angle$ Research Square \\ The association of dietary patterns with endocannabinoids levels in overweight and obese women
}

Neda Lotfi Yagin

Tabriz University of Medical Sciences

Soghra Aliasgharzadeh

Tabriz University of Medical Sciences

Fereshteh Aliasghari

Tabriz University of Medical Sciences

Reza Mahdavi ( $\square$ reza.mahdavi57@gmail.com )

Tabriz University of Medical Sciences

Samaneh Hajarzadeh

Tabriz University of Medical Sciences

Research

Keywords: dietary pattern, factor analysis, endocannabinoids, obesity, women

Posted Date: January 27th, 2020

DOI: https://doi.org/10.21203/rs.2.21904/v1

License: (9) This work is licensed under a Creative Commons Attribution 4.0 International License. Read Full License 


\section{Abstract}

Background: Higher levels of AEA and 2-AG the main arachidonic acid-derived endocannabinoids are frequently reported in overweight and obese individuals. A dietary approach to reduce endocannabinoid levels may be a safer. The relation between endocannabinoids and dietary patterns is poorly understood. This study was evaluated the AEA and 2-AG levels across dietary patterns quintiles in overweight and obese women.

Methods: In this cross sectional study 183 overweight and obese female (19-50 years old) were recruited. The participants were interviewed, the anadamide and 2-AG levels were measured and the dietary patterns were assessed using a validated semi quantitative food frequency questionnaire. To extract the dietary patterns factor analysis was applied. The association between AEA and 2-AG levels and dietary patterns was analyzed by linear regression.

Results: Three major dietary patterns including "western", "healthy" and "traditional" were extracted. After adjusting for BMI, waist circumference and fat mass higher levels of AEA and 2-AG were observed in those who were in the highest quintile of the western pattern ( $P$ for trend $<0.05$ ). Also, in both unadjusted and adjusted model, significantly lower AEA and 2-AG levels were detected in women in the highest quintile of the healthy pattern ( $P$ for trend $<0.01$ ). Also, the association between AEA and 2- AG levels and traditional pattern became significant after controlling for confounders ( $P$ for trend $<0.01$ ).

Conclusion: Regarding the lower levels of endocannabinoids in healthy dietary pattern, modification of the endocannabinoids tones by changes in diet can be a promising approach in prevention and treatment of obesity.

\section{Background}

At present, overweight/obesity is the most prevalent dietary problem, and is one of the serious public health issues in developing countries such as Iran [1,2]. The worldwide impact of overweight/obesity and its complications is indicating an urgent need to distinguish important molecular mechanisms and metabolic targets implicated in energy balance following the altered nutrition [3]. Neuroendocrine system together with autonomic nervous system circuits control food intake which is the fundamental component of normal body maintenance [4].

Moreover, in the past 15 years, the endocannabinoid system (ECS) has appeared as a lipid signaling system involved in energy balance regulation, as it has control on every aspect of calorie regulation [5]. The endocannabinoid system consists of the cannabinoid 1 and 2 receptors, endogenous ligands $\mathrm{N}$ arachidonoyl-ethanolamide (anandamide), and 2-arachidonoyl glycerol (2-AG), and enzymes responsible for the biosynthesis and degradation of ligands [6]. The endogenous ligands are lipid derivatives of a $\omega$ 6-polyunsaturated fatty acid, arachidonic acid, with multiple functions [7]. A growing body of evidence nowadays suggests that the overstimulation of ECS can lead to obesity and obesity- associated disorders [8]. Although, the potential roles of endocannabinoids and their respond to energy balance are highly 
recognized; the important effect of different kinds of diets on endocannabinoids levels are still largely unknown [9]. Dietary intake of fatty acids and PUFAs is regarded as the main source of the endogenous cannabinoids biosynthesis in mammals, and changes in nutritional status might impact the levels of EC [10]. However, aside from the effect of PUFA and other fatty acids on levels of endocannabinoids, the impact of other nutrients on them is ambiguous.

On the other hand, nutrition transition specifically acquisition of a western diet (large amounts of red meats, fast foods and snacks) is one of the factors which may help explain the changes in diet as well as obesity and associated disorders [7]. Recently researchers have focused on dietary patterns for evaluating the diet-diseases relationship $[11,12]$. It is an approach with more precise diet assessment which provides more detailed data than analyzing one nutrient or foods as they are usually consumed together [13].

Higher levels of ARA-derived AEA and 2-AG are frequently seen in overweight and obese individuals, and to the best of our knowledge there is no reported study conducted on assessing the association between endocannabinoids levels and overall dietary patterns in overweight/ obese females. We carried out this study to examine endocannabinoids levels with dietary patterns in overweight and obese women.

\section{Materials And Methods Study participants}

This cross-sectional study was carried out on 183 overweight and obese women during October 2017 to February 2018, who lived in Tabriz. Women with age between 19-50 years and BMI between 25 to $40 \mathrm{~kg} / \mathrm{m}^{2}$ were recruited through announcements and flyer distribution in health care centers (Table 1). The subjects free of any chronic disease such as diabetes, kidney and liver disease were included in the study. Additionally, being pregnant or lactating, consumption of any medicine affecting appetite such as antidepressant drugs as well as steroids, significant weight loss during last three months were also the exclusion criteria.

The study protocol was approved by the Ethics Committee of Tabriz University of Medical Science (IR.TBZMED.REC.1396.620). A comprehensible consent form was signed by each participant.

\section{Anthropometric and biochemical measurements}

Weight of each participants was determined in fasted state wearing light clothe and shoeless with a precision of $0.1 \mathrm{~kg}$. Height was measured by a stadiometer in standing position on barefoot. Body mass index calculated as weight $(\mathrm{kg}) /\left(\right.$ height $\left.(\mathrm{cm})^{2}\right)$.

Whole blood samples were collected at baseline, after a $14 \mathrm{~h}$ fasting and the serum and plasma samples were separated from whole blood by centrifugation at $1500 \mathrm{~g}$ at $4^{\circ} \mathrm{C}$ for $10 \mathrm{~min}$ and were frozen immediately at $-80^{\circ} \mathrm{C}$ until assay. Plasma samples were processed no later than 10 min. Analyze of AEA 
and 2-AG circulating levels were done using human enzyme-linked immunosorbent assay (ELISA) kits (HANGZHOU EASTBIOPHARM CO., LTD.).

\section{Dietary assessment}

Participants' dietary intake was determined applying a valid and reliable semi-quantitative food frequency questionnaire (FFQ) containing 147 food items (with standard portion sizes) consumed frequently by Iranians. The questionnaire was completed via direct interview. The women were asked to report the frequency of consumption of every food item on a daily, weekly, monthly or yearly basis. Afterward, the stated frequency for each food object was converted into a daily intake. By utilizing household measures the serving sizes of the consumed meals were transformed into grams. After computing the energy intake from all meal items, overall energy intake of each participant was calculated. Regarding the likeness of nutrient profile, each of the 147 food items were allocated to one of the 31 specified food groups (Table 2). Standard methods were applied by trained researchers for measurements of the anthropometric indices.

\section{Statistical analysis}

Data were analyzed using SPSS software (SPSS Inc., Chicago, IL, version 20). The 147 food items in the FFQ were changed into daily consumption frequencies and were then categorized into 35 food groups, with regards to their consumption frequencies and nutritional characteristics (Table 2). Factor analysis and principal component analysis were applied to detect the major eating patterns. The food groups that had communalities $<0.3$ were excluded. Eigenvalue $>1.5$, Scree plot and natural interpretation were the criteria for retention of the factors. The extracted factors (dietary patterns) were named concerning the food groups that had high positive loading, were comparable to the healthy and western dietary patterns and also based on the existing literatures. By summing up intakes of food groups weighted by their factor loadings the factor score for each pattern was computed. And every participant received a factor score for each identified pattern. Participants were categorized by quintile of dietary pattern scores.

The association between nutritional patterns and AEA, 2-AG were analyzed using linear regression models in different models (Model1: unadjusted, Model2: adjusted for BMI, WC and fat mass). The statistical significance was considered at the $\mathrm{P}<0.05$ level.

\section{Results}

The participants' demographic, anthropometric, and laboratory data is presented in table 1 . The participants' mean age and BMI were $34.2 \pm 8.22$ years old, and $32.44 \pm 3.79 \mathrm{~kg} / \mathrm{m}^{2}$ respectively. The $55.2 \%$ of the individuals had low levels of activity, $38.8 \%$ were moderately active and the rest of them were highly active (6\%). The AEA and 2-AG levels were $4.54 \pm 1.22 \mathrm{ng} / \mathrm{ml}$ and

$5.42 \pm 1.50 \mathrm{ng} / \mathrm{ml}$ respectively. By the use of factor analysis, 3 major dietary patterns were extracted which were labeled as following: the healthy dietary pattern (high in other vegetables, Cruciferous vegetables, tomato, yellow vegetables, low fat dairy, green leafy vegetable, and red meat), the western 
dietary pattern (high in processed meat, organ meat, pizza, processed meat, coffee, sweets, soft drinks and French fries), and the traditional dietary pattern (tea, fish, poultry, and sugar) (Table 3). Totally these three factors explained $25.47 \%$ of the whole variance.

Linear regression analysis was applied to assess the association of AEA and 2-AG across quintile of dietary patterns (Table 4). In the case of western dietary pattern, in unadjusted model, there was no significant relation between 2-AG and western dietary pattern $(P$ for trend $=0.09)$. However, regarding AEA those in the highest quintile of this pattern had significantly higher levels of AEA ( $P$ for trend $<0.01$ ) in comparison with those in the lowest quintile. Additionally, after controlling for $\mathrm{BMI}$, waist circumference and fat mass the $\mathrm{p}$ for trend decreased and relation between 2-AG and western dietary pattern became significant $(P$ for trend $<0.05$ ) and significantly higher levels of 2-AG were observed in those who were in the highest quintile of this pattern. Concerning the healthy dietary pattern, in unadjusted and adjusted model, women in the highest quintile of this pattern had significantly lower AEA and 2-AG levels ( $P$ for trend $<0.01)$ compared to those in the lowest quintile. Although, there was not any significant association between traditional pattern and AEA and 2- AG levels ( $P$ for trend $>0.05$ ), after adjusting for BMI, waist circumference and fat mass the relation became significant $(P$ for trend $<0.01)$.

\section{Discussion}

It has been proposed that diets high in fat are have the potential of modulating endocannabinoids levels irrespective of their FA composition [14-16]. Regarding the dietary intake as one of the important factors in determining of the endocannabinoids tones, it appears that complete investigation to identify dietary patterns can be helpful in understanding the association of diet and AEA, 2-AG levels. To the best of our knowledge, this study was the first study which has evaluated the association of dietary patterns with endocannabinoids levels in overweight/obese women.

In the current study, three major dietary patterns were identified in in overweight/obese women: the "western", the "healthy", and traditional dietary patterns. Our results were in line with those of Esmaillzadeh and colleagues who identified three major dietary patterns among 486 obese women: healthy, western, and Iranian dietary pattern [17]. Also, in nurses with premenstrual syndrome and female with metabolic syndrome three major dietary patterns including "western", "healthy", and "traditional" patterns were extracted $[18,19]$. Additionally, in a rather large sample size (1433 Iranian adults), western, semi healthy and healthy fat patterns were identified [20]. However, in a study by Hu et al. among men aged 40-75 years two major patterns: the "western" pattern (high intakes of processed meat, red meat, French fries, high-fat dairy products, refined grains, sweets, and dessert) and the "prudent" pattern (high intakes of whole grains, legumes, vegetables, fruit, fish, and poultry) were reported [21]. Agodi et al., derived two major dietary patterns, the first one named western which was high in hot-dog, hamburger, processed meat, fries, white bread, and salty snacks. On the contrary, the other dietary pattern named prudent was specified by high intake of nuts, cereals, cooked and raw vegetables, fruit, fish, jam and honey [22]. Compared to previous studies, the observed differences in our findings might stem from the 
different types of the diseases, the number of food items in used FFQ and differences in geographic areas of conducted studies.

In present study, high adherence to western dietary pattern resulted in significantly higher levels of AEA and 2-AG compared to the high adherence to healthy dietary pattern. The positive association between the western pattern and endocannabinoids levels could be due to the food groups components found on this dietary pattern. In this pattern organ meat, processed meat, pizza, French fries, soft drinks were dominant. There is notable shift to western dietary pattern consumption greatly loaded in red meats, fast foods, and soft drinks in developing countries such as Iran [20]. Moreover, the prevalence of high fat diets ( $~ 40 \%$ of energy) is rising globally due to their palatability and also the fats low cost $[23,24]$. ECS are lipid mediators and their biosynthesis can be modified directly by dietary fat intake.

In animals, diets high in fat prompt binge eating behaviors [25] and lead in significantly elevated levels of AEA and 2-AG $[26,27]$ and intestinal motility [28] which in turn can increase the stimulation of cannabinoid receptor. Also, high fat diets cause an increase in FA synthesis which is partially triggered by chronic CB1 activation and subsequent induction of expression of the lipogenic transcription factor sterol regulatory element-binding protein-1c (SREBP-1c), and greater production of acetyl coenzyme-A carboxylase- 1 and fatty acid synthase production [27]. As a result, the fatty acid biosynthetic pathway might indicate a common molecular target for the central appetitive and peripheral metabolic effects of endocannabinoids. Also, a decrease in MGL and FAAH activity and an increase in NAPE-PLD action have been found to cause an elevation in AEA and 2-AG levels in response to high fat diets in animals [29].

Additionally, since de novo synthesis of $\omega-6$ and $\omega-3$ fatty acids is not possible, the dietary intake of these fatty acids is reflected in the tissues fatty acid composition [30]. A high dietary intake of linoleic acid $(\omega-6)$ can raise the arachidonic acid synthesis triggering EC production [31]. Elevated levels of 2-AG in the whole brain and plasma of adults and developing animals was observed in rats deficient in $\omega-3$; whereas, supplementation with $\omega-3$ seems to decrease AEA levels [32,33]. Furthermore, Alvheim et al., showed that in a diet with $60 \%$ of energy from lipids rising energy from linoleic acid from $1-8 \%$ led to an elevation in AA in the red blood cells and liver and a subsequent 3-fold increase in both AEA and 2-AG [34].

Researchers have largely focused on the macronutrient portions rather than particular FA intakes. We evaluated the association of dietary patterns with AEA and 2-AG levels which can be regarded as the main strength point of present study.

In light of some limitations the presented findings should be interpreted: the cross-sectional design, which makes it impossible to demonstrate the causality of the interactions. A FFQ with standard portion sizes was applied to estimate the food intakes in which the measurement error might not be precluded and may contain inaccuracies.

\section{Conclusions}


In conclusion, the manipulation of dietary FA, adapting healthy life style along with reduction in $\omega-6$ to $\omega-3$ ratio by modification of endocannabinoids tones can have promising results towards prevention and treatment of overweight and obesity.

\section{Abbreviations}

BMI

Body mass index; ECS:endocannabinoid system; Anandamide:N-arachidonoyl-ethanolamide; 2-AG:2arachidonoyl glycerol; FFQ:food frequency questionnaire;

\section{Declarations}

\section{Acknowledgments}

The authors wish to thank the participants for their cooperation, time and patience and Tabriz University of Medical Sciences for the financial support. The results of this paper are from Neda Lotfi's Ph.D. thesis.

\section{Authors' contributions}

$\mathrm{NLY}$ and RM was the major contributor of the manuscript, designed the research project and agreed for all aspects of the work and wrote the manuscript. SA and SH collected and interpreted the data and performed the statistical analysis. FA made substantial contributions to the data interpretation. All authors read and approved the final version of manuscript.

\section{Funding}

This study was funded Tabriz University of Medical Sciences.

\section{Availability of data and materials}

The datasets used and/or analyzed during the current study are available from the corresponding author on reasonable request.

\section{Ethics approval and consent to participate}

All participants provided written informed consent prior to commencing the study. The study and subsequent analysis were approved by the Ethics Committee of Tabriz University of Medical Sciences. 


\section{Competing interests}

The authors declare that there is no conflict of interest.

\section{Consent for publication}

Not applicable

\section{References}

1. Hu L, Huang X, You C, Li J, Hong K, Li P, et al. Prevalence of overweight, obesity, abdominal obesity and obesity-related risk factors in southern China. PloS one. 2017;12(9):e0183934-e.

2. Tabrizi JS, Sadeghi-Bazargani H, Farahbakhsh M, Nikniaz L, Nikniaz Z. Prevalence and Associated Factors of Overweight or Obesity and Abdominal Obesity in Iranian Population: A Population-based Study of Northwestern Iran. Iran J Public Health. 2018;47(10):1583-1592.

3. Ghanemi A, Yoshioka M, St-Amand J. Broken Energy Homeostasis and Obesity Pathogenesis: The Surrounding Concepts. J Clin Med. 2018;7(11):453.

4. Lenard NR, Berthoud H-R. Central and peripheral regulation of food intake and physical activity: pathways and genes. Obesity. 2008;16 Suppl 3(Suppl 3):S11-S22.

5. Gatta-Cherifi B, Cota D. New insights on the role of the endocannabinoid system in the regulation of energy balance. Int J Obes (Lond). 2016;40(2):210-219.

6. Collu R, Scherma M, Piscitelli F, Giunti E, Satta V, Castelli MP, et al. Impaired brain endocannabinoid tone in the activity-based model of anorexia nervosa. Int J Eat Disord. 2019;52(11):1251-1262.

7. Freitas HR, Isaac AR, Malcher-Lopes R, Diaz BL, Trevenzoli IH, De Melo Reis RA. Polyunsaturated fatty acids and endocannabinoids in health and disease. Nutr Neurosci. 2018;21(10):695-714.

8. Yagin NL, Aliasgari F, Aliasgharzadeh S, Mahdavi R, Akbarzadeh M. The influence of the fatty acid amide hydrolase $385 \mathrm{C}>\mathrm{A}$ single nucleotide polymorphisms on obesity susceptibility. Mol Biol Rep. 2019;46(5):5049-5055.

9. Naughton SS, Mathai ML, Hryciw DH, McAinch AJ. Fatty Acid Modulation of the Endocannabinoid System and the Effect on Food Intake and Metabolism. Int J Endocrinol. 2013;2013:11.

10. Watkins BA, Kim J. The endocannabinoid system: directing eating behavior and macronutrient metabolism. Front Psychol. 2015;5:1506-.

11. Hu FB. Dietary pattern analysis: a new direction in nutritional epidemiology. Curr Opin Lipidol. 2002;13(1):3-9.

12. Kant AK. Dietary patterns and health outcomes. J Am Diet Assoc. 2004;104(4):615-635.

13. Shim J-S, Oh K, Kim HC. Dietary assessment methods in epidemiologic studies. Epidemiol Health. 2014;36:e2014009-e. 
14. Diep TA, Madsen AN, Holst B, Kristiansen MM, Wellner N, Hansen SH, et al. Dietary fat decreases intestinal levels of the anorectic lipids through a fat sensor. The FASEB J. 2011;25(2):765-774.

15. Di Marzo V, Capasso R, Matias I, Aviello G, Petrosino S, Borrelli F, et al. The role of endocannabinoids in the regulation of gastric emptying: alterations in mice fed a high-fat diet. Br J Pharmacol. 2008;153(6):1272-1280.

16. DiPatrizio NV, Astarita G, Schwartz G, Li X, Piomelli D. Endocannabinoid signal in the gut controls dietary fat intake. Proc Natl Acad Sci U S A. 2011;108(31):12904-12908.

17. Esmaillzadeh A, Azadbakht L. Major dietary patterns in relation to general obesity and central adiposity among Iranian women. J Nutr. 2008;138(2):358-363.

18. Esmaillzadeh A, Kimiagar M, Mehrabi Y, Azadbakht L, Hu FB, Willett WC. Dietary patterns, insulin resistance, and prevalence of the metabolic syndrome in women. Am J Clin Nutr. 2007;85(3):910918.

19. Farasati N, Siassi F, Koohdani F, Qorbani M, Abashzadeh K, Sotoudeh G. Western dietary pattern is related to premenstrual syndrome: a case-control study. Br J Nutr 2015;114(12):2016-2021.

20. Zaribaf F, Mohammadifard N, Sarrafzadegan N, Karimi G, Gholampour A, Azadbakht L. Dietary patterns in relation to lipid profiles among Iranian adults. J Cardiovasc Thorac Res. 2019;11(1):1927.

21. Hu FB, Rimm EB, Stampfer MJ, Ascherio A, Spiegelman D, Willett WC. Prospective study of major dietary patterns and risk of coronary heart disease in men. Am J Clin Nutr. 2000;72(4):912-921.

22. Agodi A, Maugeri A, Kunzova S, Sochor O, Bauerova H, Kiacova N, et al. Association of Dietary Patterns with Metabolic Syndrome: Results from the Kardiovize Brno 2030 Study. Nutrients. 2018;10(7).

23. Drewnowski A, Popkin BM. The nutrition transition: new trends in the global diet. Nutr Rev. 1997;55(2):31-43.

24. Drewnowski A, Shrager EE, Lipsky C, Stellar E, Greenwood MR. Sugar and fat: sensory and hedonic evaluation of liquid and solid foods. Physiol Behav. 1989;45(1):177-183.

25. Higuchi S, Irie K, Yamaguchi R, Katsuki M, Araki M, Ohji M, et al. Hypothalamic 2arachidonoylglycerol regulates multistage process of high-fat diet preferences. PloS one. 2012;7(6):e38609.

26. Izzo AA, Piscitelli F, Capasso R, Aviello G, Romano B, Borrelli F, et al. Peripheral endocannabinoid dysregulation in obesity: relation to intestinal motility and energy processing induced by food deprivation and re-feeding. Br J Pharmacol. 2009;158(2):451-61.

27. Osei-Hyiaman D, DePetrillo M, Pacher P, Liu J, Radaeva S, Bátkai S, et al. Endocannabinoid activation at hepatic CB1 receptors stimulates fatty acid synthesis and contributes to diet-induced obesity. J Clin Invest. 2005;115(5):1298-1305.

28. Piscitelli F, Carta G, Bisogno T, Murru E, Cordeddu L, Berge K, et al. Effect of dietary krill oil supplementation on the endocannabinoidome of metabolically relevant tissues from high-fat-fed mice. Nutr Metab. 2011;8(1):51. 
29. Aviello G, Matias I, Capasso R, Petrosino S, Borrelli F, Orlando P, et al. Inhibitory effect of the anorexic compound oleoylethanolamide on gastric emptying in control and overweight mice. J Mol Med. 2008;86(4):413-422.

30. Lands WE, Libelt B, Morris A, Kramer NC, Prewitt TE, Bowen P, et al. Maintenance of lower proportions of $(n-6)$ eicosanoid precursors in phospholipids of human plasma in response to added dietary $(n-$ 3) fatty acids. Biochim Biophys Acta. 1992;1180(2):147-162.

31. Bosma-den Boer MM, van Wetten ML, Pruimboom L. Chronic inflammatory diseases are stimulated by current lifestyle: how diet, stress levels and medication prevent our body from recovering. Nutr Metab. 2012;9(1):32.

32. Wood JT, Williams JS, Pandarinathan L, Janero DR, Lammi-Keefe CJ, Makriyannis A. Dietary docosahexaenoic acid supplementation alters select physiological endocannabinoid-system metabolites in brain and plasma. J Lipid Res. 2010;51(6):1416-1423.

33. Watanabe S, Doshi M, Hamazaki T. n-3 Polyunsaturated fatty acid (PUFA) deficiency elevates and n3 PUFA enrichment reduces brain 2-arachidonoylglycerol level in mice. Prostaglandins Leukot Essent Fatty Acids. 2003;69(1):51-59.

34. Alvheim AR, Malde MK, Osei-Hyiaman D, Lin YH, Pawlosky RJ, Madsen L, et al. Dietary linoleic acid elevates endogenous 2-AG and anandamide and induces obesity. Obesity. 2012;20(10):1984-1994.

\section{Tables}




\begin{tabular}{|ll|}
\hline \multicolumn{2}{|l|}{ Table 1. Participants' demographic, anthropometric, and laboratory data } \\
\hline Variables & Mean \pm SD \\
\hline Age (year) & $34.23 \pm 8.22$ \\
Weight $(\mathrm{kg})$ & $83.12 \pm 11.43$ \\
\hline Height $(\mathrm{cm})$ & $159.93 \pm 5.57$ \\
\hline BMl $\left(\mathrm{kg} / \mathrm{m}^{2}\right)$ & $32.44 \pm 3.79$ \\
\hline Waist Circumference $(\mathrm{cm})$ & $101.13 \pm 9.04$ \\
\hline Fat mass (kg) & $34.13 \pm 7.82$ \\
\hline AEA (ng/ml) & $4.54 \pm 1.22$ \\
\hline 2- AG (ng/ml) & $5.42 \pm 1.50$ \\
\hline Physical activity levels & $\mathrm{n}(\%)$ \\
\hline Low & $101(55.2 \%)$ \\
\hline Moderate & $71(38.8 \%)$ \\
\hline High & $11(6 \%)$ \\
\hline All data are mean (SD) or percentage of participants; Abbreviations: $\mathrm{BMI}:$ body mass index; AEA: \\
\hline Anandamide; 2-AG: 2-arachidonoylglycerol. \\
\hline
\end{tabular}




\section{Table 2. Food groups using for dietary pattern analysis}

Food groups* Food items

$\begin{array}{ll}\text { Processed meat } & \text { Sausages } \\ \text { Red meat } & \text { Beef, lamb, burgers }\end{array}$

Organ meat Brain, liver, heart, kidney, tripe, offal

Fish All types of fish, canned tuna

Poultry Chicken

Egg Eggs

Low fat dairy Low fat milk, yoghurt, dough, curd

High fat dairy High fat milk, full fat yoghurt, chocolate milk, cheese, ice cream, clotted cream

Fruits Different kind of fresh fruits, dry fruits, fruit conserves

Cruciferous

All types of Cruciferous vegetables

vegetables

Yellow vegetables Cooked and raw carrots, pumpkin.

Green leafy vegetable Lettuce, Spinach

Other vegetables All other type of vegetables

Tomato Tomato

Legumes Different kind of beans, peas, lentil

Potato Cooked potato

French fries $\quad$ French fries

Whole grains Dark Iranian bread including barbari, sangak, taftoon, barely

Refined grains White bread including lavash, baguette, rice, pasta, vermicelli

Snacks Biscuits, crackers, chips, cheese snacks

Nuts

Roasted and salted Walnuts, peanuts, hazelnuts, pistachio, almonds, seeds

Solid oils

Hydrogenated vegetable oil, animal oil, margarine, butter, mayonnaise

Olive

Olive oil, olive

Liquid oil

All other type of liquid oils 
Table 2. Food groups using for dietary pattern analysis

Vegetable pickles

Pastries

Sugars

Tea

Coffee

Pizza

Natural fruit juice

Soft drinks
All type of vegetable pickles

All types of cookies, cakes and pastries

Different kinds of transitional sweets, candies, honey, jam, marmalade Tea

Coffee

All types of Pizza

All types of natural fruit juices

All types of cola drinks, industrial juice

*31 food groups based on similarity of nutrient profile.

\section{Table 3. Factor loading matrix for major dietary patterns ${ }^{1}$}

\begin{tabular}{|llll|}
\hline Food groups & Western pattern & Healthy pattern & Traditional pattern \\
\hline $\begin{array}{l}\text { Organ meat } \\
\text { Pizza }\end{array}$ & 0.80 & - & - \\
\hline Processed meat & 0.75 & - & - \\
\hline Coffee & 0.69 & - & - \\
\hline Sweets & 0.65 & - & - \\
Soft drinks & 0.44 & - & - \\
French fries & 0.41 & - & - \\
Other vegetables & 0.39 & - & - \\
Cruciferous vegetables & - & 0.77 & - \\
Tomato & - & 0.70 & - \\
Yellow vegetables & - & 0.68 & - \\
Low fat dairy & - & 0.56 & - \\
Green leafy vegetable & - & 0.52 & - \\
Red meat & - & 0.31 & 0.67 \\
Tea & - & - & 0.61 \\
Fish & - & - & 0.57 \\
Sougar & - & - & 6.33 \\
Percentage of variance explained (\%) & 9.85 & 9.30 & \\
1Values $<0.3$ were excluded for simplicity. & & \\
\hline
\end{tabular}


Table 4. Association between AEA and 2-AG with dietary patterns quintiles

Models Dietary pattern quintile Western pattern - Healthy pattern

Traditional pattern

AEA

Model

\begin{tabular}{ll} 
Quintile 1 & reference \\
\hline Quintile 2 & $0.35[-0.2-0.91]$ \\
\hline Quintile 3 & $0.16[-0.39-0.72]$ \\
\hline Quintile 4 & $0.45[-0.1-1.01]$ \\
\hline Quintile 5 & $0.93[0.37-1.49]$ \\
\hline P value for trend & $<0.01$
\end{tabular}

reference

reference

$-0.24[-0.76-0.28] \quad 0.15[-0.42-0.73]$

Quintile 1

reference

$-0.73[-1.26-(-0.21)]$

$0.15[-0.42-0.73]$

$-1.24[-1.79-(-0.74)]$

$-0.06[-0.63-0.51]$

Model 2

Quintile 2

$0.34[-0.17-0.86]$

$-1.26[-1.79-(-0.73)]$

$0.08[-0.49-0.66]$

Quintile 3

$0.11[-0.41-0.65]$

$<0.01$

0.94

Quintile 4

0.57 [0.04-1.11]

reference

reference

Quintile 5

0.82 [0.29-1.36]

$-0.05[-0.54-0.42]$

0.19 [-0.33-0.72]

$P$ value for trend

$<0.01$

$-0.42[-0.91-0.06]$

$0.36[-0.16-0.90]$

$-1.11[-1.59-(-0.62)]$

0.14 [-0.38-0.67]

$-1.15[-1.63-(-0.67)]$

$0.34[-0.19-0.89]$

$<0.01$

$<0.01$

2-AG

\begin{tabular}{|c|c|c|c|c|}
\hline \multirow{6}{*}{ Model 1} & Quintile 1 & reference & reference & reference \\
\hline & Quintile 2 & $0.26[-0.44-0.96]$ & $-0.56[-1.24-0.11]$ & $0.27[-0.43-0.98]$ \\
\hline & Quintile 3 & $0.18[-0.51-0.87]$ & $-1.05[-1.73-(-0.36)]$ & $-0.21[-0.93-0.49]$ \\
\hline & Quintile 4 & $-0.12[-0.81-0.57]$ & $-1.04[-1.72-(-0.36)]$ & $0.08[-0.62-0.79]$ \\
\hline & Quintile 5 & $0.87[0.17-1.57]$ & $-1.38[-2.06-(-0.69)]$ & $-0.16[-0.88-0.54]$ \\
\hline & $P$ value for trend & 0.09 & $<0.01$ & 0.51 \\
\hline \multirow[t]{6}{*}{ Model 2} & Quintile 1 & reference & reference & reference \\
\hline & Quintile 2 & $0.26[-0.26-0.79]$ & $-0.18[-0.69-0.33]$ & $0.32[-0.21-0.86]$ \\
\hline & Quintile 3 & $0.14[-0.40-0.69]$ & $-0.41[-0.93-0.10]$ & $0.17[-0.36-0.71]$ \\
\hline & Quintile 4 & $0.19[-0.35-0.75]$ & $-0.62[-1.13-(-0.11)]$ & $0.44[-0.09-0.98]$ \\
\hline & Quintile 5 & $0.68[0.13-1.23]$ & $-1.14[-1.65-(-0.64)]$ & $0.28[-0.25-0.83]$ \\
\hline & $P$ value for trend & $<0.05$ & $<0.01$ & $<0.01$ \\
\hline
\end{tabular}




\section{Table 4. Association between AEA and 2-AG with dietary patterns quintiles}

Model 1: Unadjusted.

Model 2: adjusted for BMI, WC and fat mass 